\title{
Evaluation of the Caries Removal Efficacy of Several Organic Acid Reagents
}

\author{
Yoshishige Yamada, ${ }^{1, *}$, Yuichi Kimura² and Mozammal Hossain ${ }^{3}$ \\ ${ }^{1}$ Division of Operative Dentistry, Department of Conservative Dentistry, Ohu University School of Dentistry, Fukushima, Japan \\ ${ }^{2}$ Division of Endodontics, Department of Conservative Dentistry, Ohu University School of Dentistry, Fukushima, Japan \\ ${ }^{3}$ Department of Conservative Dentistry and Endodontics, Bangabandhu Sheikh Mujib Medical University, Faculty of Dentistry, Dhaka, Bangladesh
}

*Corresponding author: Yoshishige Yamada, Division of Operative Dentistry, Department of Conservative Dentistry, Ohu University School of Dentistry, 31-1 Misumido, Tomita-machi, Koriyama-city, Fukushima 963-8611, Japan, E-mail: y-yamada@den.ohu-u.ac.jp

Received: 22 Apr, 2021 | Accepted: 15 May, 2021 | Published: 21 May, 2021

Citation: Yamada Y, Kimura Y, Hossain M (2021) Evaluation of the Caries Removal Efficacy of Several Organic Acid Reagents. Int J Dent Oral Health 7(4): dx.doi.org/10.16966/2378-7090.365

Copyright: (C) 2021 Yamada Y, et al. This is an open-access article distributed under the terms of the Creative Commons Attribution License, which permits unrestricted use, distribution, and reproduction in any medium, provided the original author and source are credited.

\begin{abstract}
Purpose: Chemo-mechanical techniques are considered as a minimal intervention in caries removal. The purpose of this study was to evaluate the effectiveness of several organic acid reagents in caries removal.

Materials and Methods: Twenty-five human permanent teeth with caries at the cervical portion were removed by four organic acid reagents (10\% of citric acid, tartaric acid, malic acid, malonic acid) and Carisolv. The treatment time was measured followed by examined the cavity floors by stereoscope and scanning electric microscope (SEM).

Results: The average caries removal times with $10 \%$ citric acid, tartaric acid, malic acid, malonic acid and Carisolv treatment were 8.44 min \pm 0.67 , $9.12 \mathrm{~min} \pm 0.96,11.40 \mathrm{~min} \pm 0.34,12.20 \mathrm{~min} \pm 0.46$ and $11.16 \mathrm{~min} \pm 0.63$, respectively.

The same irregular surface condition was observed by stereoscope in all specimens. In SEM observation, the surface after $10 \%$ citric acid reagent, tartaric acid reagent and Carisolv treatment revealed many areas where the smear layer was removed and dentinal tubules were exposed. In contrast, the cavity surface was completely covered with debris like a smear layer after $10 \%$ malic acid and malonic acid treatment.
\end{abstract}

Conclusion: Based on treatment time and smear layer removal, citric acid and tartaric acid had almost the same level of caries removal effect. They seem to be very useful for carious dentin removal and might be good candidates for a new chemo-mechanical caries removal agent.

Keywords: Citric acid; Tartaric acid; Malic acid; Malonic acid; Carisolv; Chemo-mechanical caries removal

\section{Introduction}

Dental caries are one of the most prevalent oral diseases. Carious enamel and dentin are generally removed by low-speed rotary instruments with stainless burs. Although these conventional methods for carious dentin removal are easy and less time-consuming, there is the risk of removal of excessive tooth structure including health sound dentin. Moreover, pressure and heat during treatment with a high-speed preparative instrument may cause pain and is sometimes capable of causing dental pulpitis eventually. To avoid this problem, the chemo-mechanical caries removal method was established a few decades ago [1-6]. Carisolv [7-9] and Papacarie [10-12] have already been in the market for few years. Carisolv mainly consists of $1.0 \%$ sodium hypochlorite and three types of amino acids (glutamic acid, leucine, lysine). Papacarie consists of papain that is an enzyme seen in papaya. These agents soften carious dentin selectively; as a result, carious dentin can be removed easily. Although this method seems to be very useful in terms of minimum intervention (MI), these chemo-mechanical agents require a long treatment time and are not cost-effective. Especially, treatment time is the most important factor to be considered in general dental treatment. However, several researchers reported that the caries removal time of Carisolv [1315] and Papacarie [16] was longer than when using conventional rotary instruments. Therefore, it would be more desirable to find a new chemo-mechanical agent with better cost-effectiveness and less working time. Some organic acids such as citric acid and malic acid have been used in detergents and toothpastes as a scavenger of the organic deposited. Few organic acids might also be effective in removing caries dentin similar to Carisolv and Papacarie. The purpose of this study was to examine the ability of organic acid reagents to remove carious dentin and analyze the applicability of these organic acid reagents for chemo-mechanical caries removal.

\section{Materials and Methods}

\section{Preparation of samples and reagents}

Twenty-five human permanent teeth with caries at the cervical portion, which were extracted for orthodontic and periodontal reasons with the consent of the patients, were used in the study. All caries with similar conditions were selected; therefore prior to 
treatment, each carious dentin was analyzed according to the color, hardness of the lesion, and by dental radiography.

The teeth were brushed and washed with distilled water at room temperature. Four prototype reagents were specially prepared for this study. Reagent 1 (R1) consisted of $10 \%$ citric acid. Reagent 2 (R2) consisted of $10 \%$ tartaric acid, Reagent 3 (R3) consisted of $10 \%$ malic acid and Reagent 4 (R4) consisted of 10\% malonic acid. Other constituents included xanthan gum, and acid red was included as a thickener and dying agent in each prototype reagent, respectively. All reagents were originally prepared for this study. Each acid powder (used for chemical research) were diluted to $10 \%$ concentration with purified water and then used for this research.

\section{Experimental procedures}

Table 1 and table 2 showed the grouping of the sample, and the composition and $\mathrm{pH}$ of each reagent used for this study, respectively. All teeth were divided into 5 groups of 5 teeth each and express in the table. Group 1 to group 4 (G1 to G4) used reagents R1 to R4 (experimental groups) and group 5 which used Carisolv served as a control group (G5). The schema of the experimental procedure shows in figure 1.

Four experimental reagents ( $\mathrm{R} 1$ to $\mathrm{R} 4$ ) and Carisolv were applied for 30 seconds to each carious lesion in all groups. After the reagents and Carisolv were applied, carious dentin was gently removed with a scraping technique by using a hand excavator and the cavity was

Table 1: Grouping of the sample.

\begin{tabular}{|l|l|c|}
\hline \multicolumn{1}{|c|}{ Group } & \multicolumn{1}{c|}{ Reagent used } & Application time (s) \\
\hline G1 (experimental) $(n=5)$ & R1: $10 \%$ citric acid group & $30 \mathrm{~s}$ \\
\hline G2 (experimental) $(n=5)$ & R2: $10 \%$ tartaric acid & $30 \mathrm{~s}$ \\
\hline G3 (experimental) $(n=5)$ & R3: $10 \%$ malic acid & $30 \mathrm{~s}$ \\
\hline G4 (experimental) $(n=5)$ & R4: $10 \%$ malonic acid & $30 \mathrm{~s}$ \\
\hline G5 (Control) $(n-5)$ & Carisolv & $30 \mathrm{~s}$ \\
\hline
\end{tabular}

Table 2: Main ingredients and $\mathrm{pH}$ of each reagent.

\begin{tabular}{|c|c|c|}
\hline Reagent & Main ingredients & $\mathrm{pH}$ \\
\hline \multirow{4}{*}{ R1 } & Citric acid (10\%) & \multirow{4}{*}{1.5} \\
\hline & Xanthan gum & \\
\hline & Acid red & \\
\hline & Pure water & \\
\hline \multirow{4}{*}{ R2 } & Tartaric acid (10\%) & \multirow{4}{*}{1.5} \\
\hline & Xanthan gum & \\
\hline & Acid red & \\
\hline & Pure water & \\
\hline \multirow{4}{*}{ R3 } & Malic acid (10\%) & \multirow{4}{*}{1.7} \\
\hline & Xanthan gum & \\
\hline & Acid red & \\
\hline & Pure water & \\
\hline \multirow{4}{*}{ R4 } & Malonic acid (10\%) & \multirow{4}{*}{1.4} \\
\hline & Xanthan gum & \\
\hline & Acid red & \\
\hline & Pure water & \\
\hline
\end{tabular}

then rinsed with water spray. The caries removal was verified using a caries-detecting dye (Caries check; Nippon Shika Yakuhin Co, Ltd, Yamaguchi, Japan). The time required for caries removal was measured in all teeth.

After carious dentin removal was completed, the cavity floor was observed to verify the surface characteristics using a stereoscope (SMZ-10, Nikon, and Tokyo, Japan). All specimens were dehydrated by a graded series of ethanol, dried to a critical point with $\mathrm{CO}_{2}$, and mounted on aluminum stubs. They were sputter-coated with platinum at the thickness of $15 \mu \mathrm{m}$ and then subjected to scanning electric microscope (SEM) examination (JSMZ-T220A, JEOL, Tokyo, Japan) for morphological evaluation.

\section{Statistical analysis}

Statistical analysis of the data was performed using the MannWhitney $\mathrm{U}$ test and a value of $\mathrm{p}<0.05$ was considered significant.

\section{Results}

\section{Required caries removal time}

The time required for caries removal is shown in figure 1 . The mean \pm SD time required for G1, G2, G3, G4 and G5 were $8.44 \mathrm{~min} \pm 0.67$, $9.12 \mathrm{~min} \pm 0.96,11.40 \mathrm{~min} \pm 0.34,12.20 \mathrm{~min} \pm 0.46$ and $11.16 \mathrm{~min}$ \pm 0.63 , respectively. Meantime required for carious dentin removal by both $10 \%$ citric acid and tartaric acid reagents was statistically less than the other two organic acid reagents (10\% malic acid and malonic acid) and Carisolv $(\mathrm{p}<0.05)$ (Figure 2$)$.

\section{Surface conditions by stereoscopic observation}

The cavity surface after carious dentin removal appeared rough and irregular in all specimens. However, no specific problems such as fracture or hole were observed in any group (Figure 3). An illustration of a typical surface feature was showed in figure 4 .

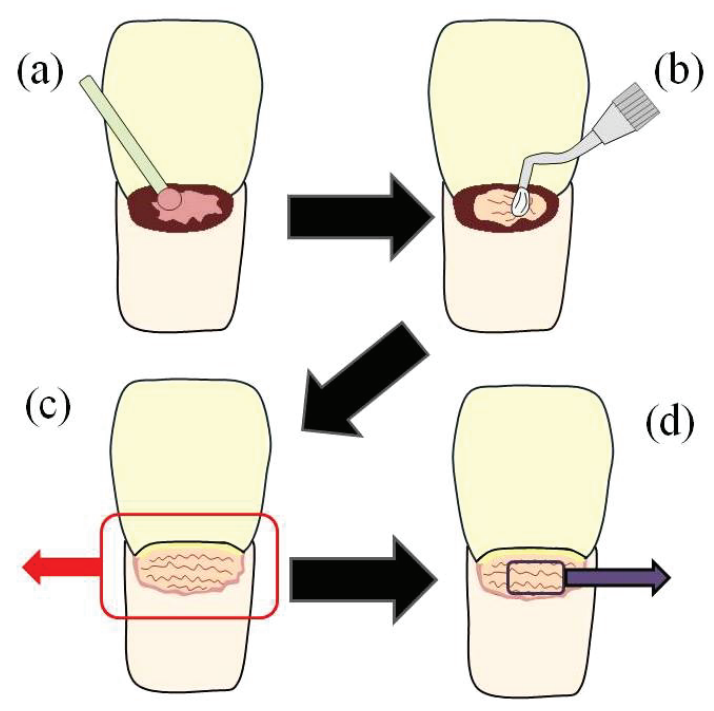

Figure 1: The schema of experimental procedure.

(a) Apply each reagent on the carious dentin and stored for 30 seconds.

(b) Removal carious dentin using hand excavator.

(c) Observation cavity surface (Red frame area) with stereoscope.

(d) SEM observation (Purple frame area). 


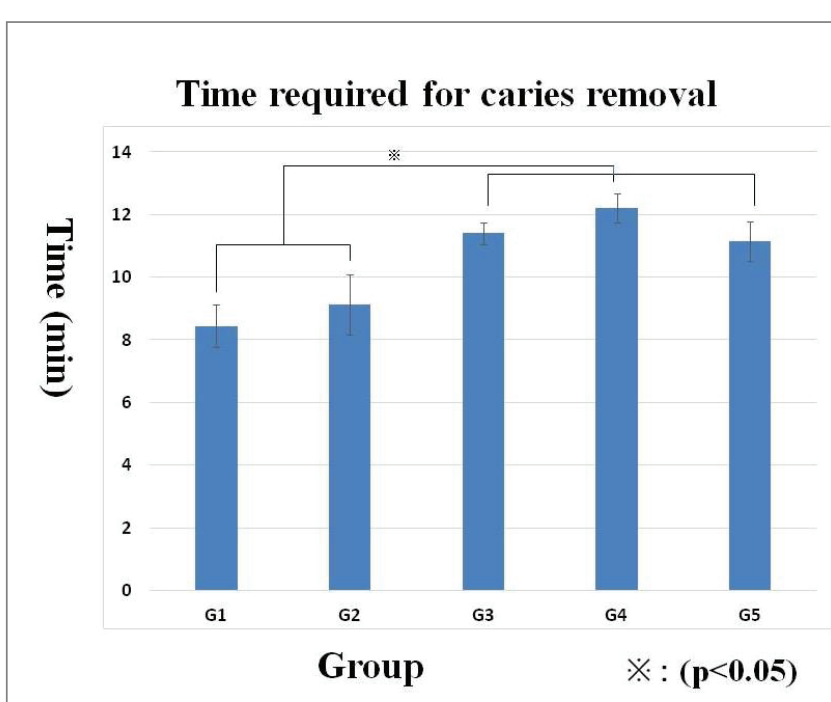

Figure 2: Time required for caries removal in each group.

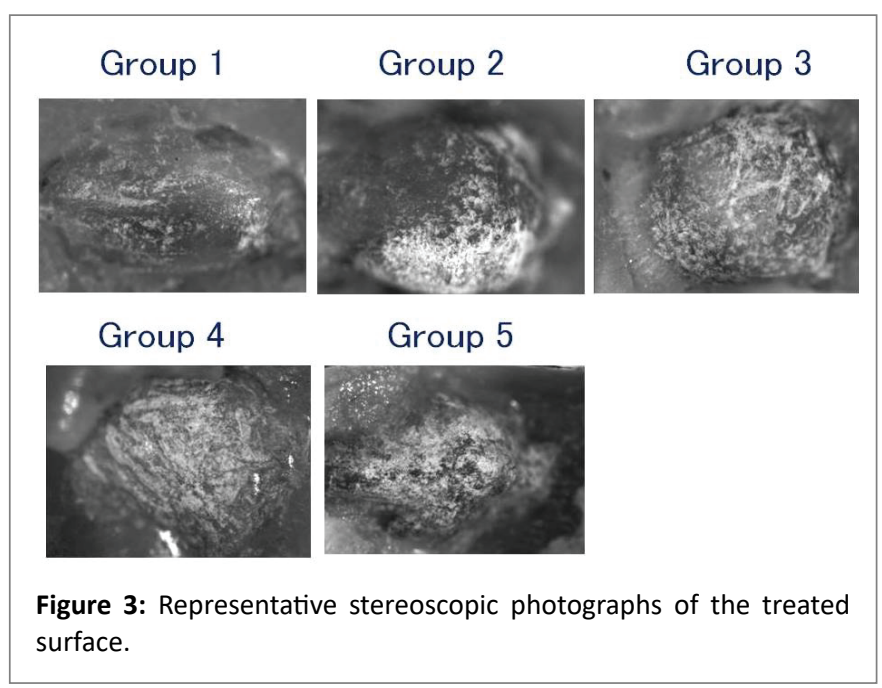

\section{SEM observation}

SEM observation revealed that the cavity surface was completely covered with debris similar to a smear layer in G3 and G4. In contrast, in G1, G2 and G5 the smear layer was removed in many areas and dentinal tubules were exposed in those areas. However, the smear layer remained in a few areas and dentinal tubules were covered (Figure 5).

\section{Discussion}

To evaluate the possibility of caries removal by acidic reagents, several acids were chosen in this study. Citric acid, tartaric acid, malic acid and malonic acid are all organic acids found in lemon, grape, apple and melon, respectively and they are known to effectively remove debris. Especially, citric acid has been used as an etching agent for composite resin filling. These four acids were uniformly used at a concentration of $10 \%$ in this study to standardize the experimental system for easy comparison of the acidic reagents.

This study demonstrated the possibility of caries removal by several organic acids. The average caries removal time of malic acid and malonic acid was around 12 minutes and almost similar to that of Carisolv treatment. While the required time for caries removal using

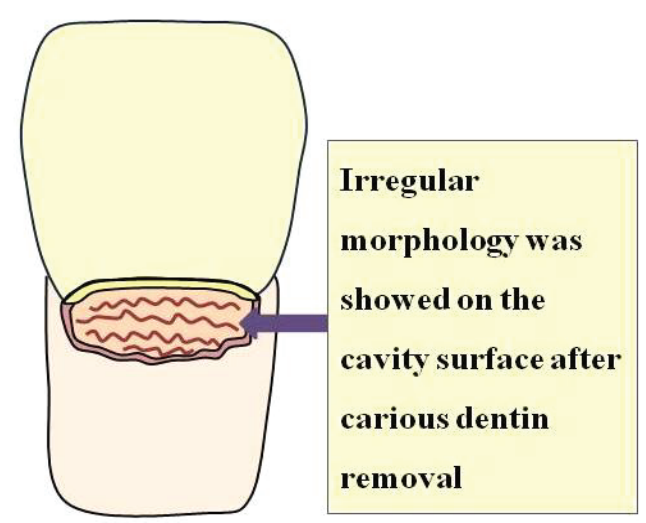

Figure 4: Illustration of standard feature of cavity surface after carious dentin removal using hand instrument.

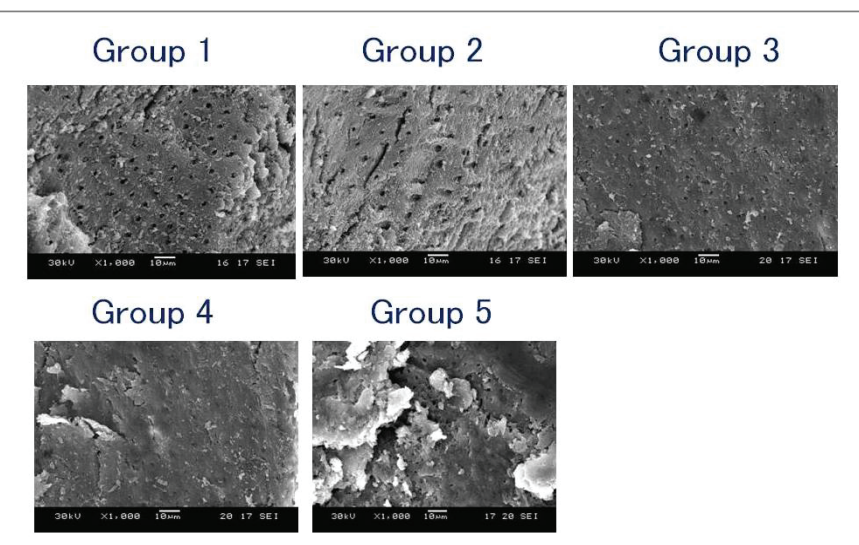

Figure 5: Representative photographs of the treated surface examined by the SEM.

$10 \%$ of citric acid and $10 \%$ of tartaric acid was around 9 minutes, it was significantly less than the other two reagents and Carisolv. In terms of treatment time, citric acid and tartaric acid have almost the same caries removal effect and seem to be very useful for carious dentin removal. From the stereoscopic observation, the cavity floor surface appeared irregular in all groups as previously described [17]. This irregular surface may have occurred as a result of scraping the area with an excavator to remove carious dentin. This rough cavity surface shows similar features to caries removal cavity surfaces with hand instruments such as spoon excavator without using any caries removal agents. Previous studies demonstrated that the adhesion between dentin surface and composite resin was not influenced by these irregular surfaces when using chemo-mechanical caries removal reagent [18-20]. Furthermore, there were no problems such as cracks or holes on any cavity floor. It indicated that the organic acid reagent did not affect sound dentin. SEM observation also revealed that the cavity surface was completely covered by organic debris like smear layer after $10 \%$ of malic acid and malonic acid reagent treatment. On the other hand, the cavity surface treated with $10 \%$ citric acid, tartaric acid reagents and Carisolv showed smear layer removal and open microtubules. It can assume that the removal of the smear layer and opening of the dentinal tubules might facilitate the adhesion between the restorative material and the dentin surface. Although previous 
papers reported smear layer removal and open dentin microtubules on the cavity surface after Carisolv treatment [20-22], this paper demonstrated that the smear layer was not removed completely in many areas of the cavity surface and most microtubules were still closed by the remaining smear layer. Similar to Carisolv treatment, the smear layer was not removed completely from the cavity surface after citric acid and tartaric acid treatment, and smear layer and closed dentin microtubules were observed in several areas of the cavity surface. Previous papers suggested that root canal irrigation with $10 \%$ of citric acid enabled efficient removal of smear layer in root canal walls [23-25]. However, both $10 \%$ citric acid reagent and $10 \%$ tartaric acid reagent could not remove the smear layer completely.

The result of the present study also revealed that the concentration of citric acid and tartaric acid used in this study might not be enough to remove the smear layer when used as a caries removal reagent. In general, EDTA application was recommended to remove the smear layer after caries removal for composite resin filling because the $\mathrm{pH}$ of EDTA was almost neutral. Therefore, it could be more safely applied to oral tissues than phosphoric acid. Furthermore, it also inhibits excessive decalcification of healthy cavity dentin. Therefore, EDTA application after caries removal with these four reagents and Carisolv might be necessary to obtain complete smear layer removal. The reason why malic acid and malonic acid were not able to remove the smear layer compared with citric acid and tartaric acid was not clear in this study. However, Kaushal R et al., also reported similar findings in the removal of smear layer using citric acid and malic acid [25]. An accurate explanation for the difference in the smear layer removing ability was not revealed in this study. The $\mathrm{pH}$ values of the four reagents, $10 \%$ of citric acid, tartaric acid, malic acid, malonic acid were $1.5,1.5,1.7$, and 1.4, respectively, and it was proposed that the difference in removal of smear layer effectiveness might not be related to $\mathrm{pH}$ of each reagent. Citric acid is known to have a chelating effect by binding free Ca ions as in EDTA [23,26,27]. Based on this evidence, the removal of the smear layer by citric acid may not be related to its $\mathrm{pH}$, but its chelating effect similar to EDTA. Recently, several researchers reported that citric acid alone or the mixture of tetracycline and acid and detergent (MTAD) have a high smear layer removing effect [28-31].

Although the mechanism by which tartaric acid removes the smear layer has not been clarified yet, it seems to be similar to the mechanical action of citric acid. Therefore, future studies are necessary to clear this question. This study showed that the smear layer could not be removed by malic acid and malonic acid. However, several reports have described the plaque removing the effect of malic acid and this acid has already been incorporated into toothpaste, presently. Haznedaroğlu $F$, et al, reported that the efficiency of removing the smear layer was improved with a high concentration of citric acid compared with a low concentration even at the same $\mathrm{pH}$ [32]. Therefore, malic acid and malonic acid may be capable of removing the smear layer when the acid concentration is increased. Further investigations should determine the optimal concentration required improving smear layer removal and consequently, caries treatment without using EDTA can be expected in the future. Moreover, few researchers reported that the cytotoxicity effects of EDTA on NIH3T3 cell (fibroblast cells isolated from mouse embryo tissue) or macrophage was higher than citric acid [33-35]. Therefore, crown restorations should be performed without using EDTA. From the results of this study, reagents with citric acid and tartaric acid were effective in caries removal, and they may serve as new chemo-mechanical caries removal agents.

\section{Conclusion}

In terms of treatment time, citric acid and tartaric acid have almost the same caries removal effect and seem to be very useful for carious dentin removal. SEM observation revealed that the cavity surface treated with $10 \%$ citric acid, tartaric acid reagents showed smear layer removal and open microtubules, while the cavity surface treated with other acidic reagents was covered by the smear layer completely. The results indicated that $10 \%$ citric acid, tartaric acid reagents might be good candidates for a new chemo-mechanical caries removal agent.

\section{Conflicts of Interest}

The authors have no conflicts of interest relevant to this article.

\section{References}

1. Gu ZQ, Chen QM, Wei S (1987) The clinical application of a chemomechanical caries removal system (Caridex): a comparative study. Compendium 8: 638-640.

2. Anusavice KJ, Kincheloe JE (1987) Comparison of pain associated with mechanical and chemomechanical removal of caries. J Dent Res 66: 1680-1683.

3. Zinck JH, McInnes-Ledoux P, Capdeboscq C, Weinberg R (1988) Chemomechanical caries removal--a clinical evaluation. J Oral Rehabil 15: 23-33.

4. Goldman M, Siu L, White RR, Kronman JA (1988) The dentinal surface of composite restorations after chemo-mechanical caries removal. J Pedod 12: 157-166.

5. Tavares M, Soparkar PM, DePaola PF (1988) Evaluation of a chemomechanical method of caries removal in root surface lesions. Quintessence Int 19: 29-32.

6. Burke FM, Lynch E (1995) Chemomechanical caries removal. J Ir Dent Assoc 41: 10-14.

7. Cederlund A, Lindskog S, Blomlöf J (1999) Effect of a chemomechanical caries removal system (Carisolv) on dentin topography of non-carious dentin. Acta Odontol Scand 57: 185-189.

8. Fure S, Lingström P, Birkhed D (2000) Evaluation of Carisolv for the chemo-mechanical removal of primary root caries in vivo. Caries Res 34: $275-280$.

9. Munshi AK, Hegde AM, Shetty PK (2001) Clinical evaluation of Carisolv in the chemico-mechanical removal of carious dentin. J Clin Pediatr Dent 26: 49-54.

10. Bussadori SK, Castro LC, Galvão AC (2005) Papain gel: a new chemomechanical caries removal agent. J Clin Pediatr Dent 30: 115-119.

11. Kotb RM, Abdella AA, El Kateb MA, Ahmed AM (2009) Clinical evaluation of Papacarie in primary teeth. J Clin Pediatr Dent 34: 117-123.

12. Bittencourt ST, Pereira JR, Rosa AW, Oliveira KS, Ghizoni JS, et al. (2010) Mineral content removal after Papacarie application in primary teeth: a quantitative analysis. J Clin Pediatr Dent 34: 229231.

13. Maragakis GM, Hahn P, Hellwig E (2001) Clinical evaluation of chemomechanical caries removal in primary molars and its acceptance by patients. Caries Res 35: 205-210.

14. Kakaboura A, Masouras C, Staikou O, Vougiouklakis G (2003) A comparative clinical study on the Carisolv caries removal method. Quintessence Int 34: 269-271.

15. Yazici $A R$, Atilla $P$, Ozgünaltay $G$, Müftüoglu $S$ (2003) In vitro comparison of the efficacy of Carisolv and conventional rotary instrument in caries removal. J Oral Rehabil 30: 1177-1182. 
16. Anegundi RT, Patil SB, Tegginmani V, Shetty SD (2012) A comparative microbiological study to assess caries excavation by conventional rotary method and a chemo-mechanical method. Contemp Clin Dent 3: 388-392.

17. Yamada $Y$, Kimura $Y$, Hossain M, Kinoshita Jl, Shimizu $Y$, et al. (2005) Caries removal with Carisolv system: criteria evaluation and microleakage test. J Clin Pediatr Dent 3: 121-126.

18. Cehreli ZC, Yazici AR, Akca T, Ozgünaltay G (2003) A morphological and micro-tensile bond strength evaluation of a single-bottle adhesive to caries-affected human dentine after four different caries removal techniques. J Dent 31: 429-435.

19. Okida RC, Martins TM, Briso AL (2007) In vitro evaluation of marginal leakage in bonded restorations, with mechanical or chemicalmechanical (Carisolv) removal of carious tissue. Braz Oral Res 21 : 176-181.

20. Li H, Wang WM, Yu SL, Wen Q (2011) Morphological and microtensile bond strength evaluation of three adhesive systems to cariesaffected human dentine with chemomechanical caries removal. J Dent 39: 332-339.

21. Correa FN, Rodrigues Filho LE, Rodrigues CR (2008) Evaluation of residual dentin after conventional and chemomechanical caries removal using SEM. J Clin Pediatr Dent 32: 115-120.

22. Zawaideh F, Palamara JE, Messer LB (2011) Bonding of resin composite to caries-affected dentin after Carisolv treatment. Pediatr Dent 33: 213-220.

23. Barroso Ldos S, Habitante SM, Jorge AO, Faria Ida S (2004) Microorganisms growth in endodontic citric-acid solutions with and without microbiological stabilizer. J Endod 30: 42-44.

24. Dewi A, Upara C, Chaiariyakul D, Louwakul P (2020) Smear Layer Removal from Root Canal Dentin and Antimicrobial Effect of Citric Acid-modified Chlorhexidine. Eur Endod J 5: 257-263.

25. Kaushal R, Bansal R, Malhan S (2020) A comparative evaluation of smear layer removal by using ethylenediamine tetraacetic acid, citric acid, and maleic acid as root canal irrigants: An in vitro scanning electron microscopic study. J Conserv Dent 23: 71-78.
26. Smith JJ, Wayman BE (1986) An evaluation of the antimicrobial effectiveness of citric acid as a root canal irrigant. J Endod 12: 54-58.

27. Yamaguchi M, Yoshida K, Suzuki R, Nakamura H (1996) Root canal irrigation with citric acid solution. J Endod 22: 27-29.

28. Mancini M, Armellin E, Casaglia A, Cerroni L, Cianconi L (2009) A comparative study of smear layer removal and erosion in apical intraradicular dentine with three irrigating solutions: a scanning electron microscopy evaluation. J Endod 35: 900-903.

29. Stojicic S, Shen Y, Qian W, Johnson B, Haapasalo M (2012) Antibacterial and smear layer removal ability of a novel irrigant, QMiX. Int Endod J 45: 363-371.

30. Rossi-Fedele G, Doğramaci EJ, Guastalli AR, Steier L, de Figueiredo JA (2012) Antagonistic interactions between sodium hypochlorite, chlorhexidine, EDTA, and citric acid. J Endod 38: 426-431.

31. Singh S, Singh M, Salgar AR, Chandrahari N, Prathibha N, et al. (2019) Time-Dependent Effect of Various Irrigants for Root Canal on Smear Layer Removal. J Pharm Bioallied Sci 11: S51-S58.

32. Haznedaroğlu F (2003) Efficacy of various concentrations of citric acid at different $\mathrm{pH}$ values for smear layer removal. Oral Surg Oral Med Oral Pathol Oral Radiol Endod 96: 340-344.

33. Sceiza MF, Daniel RL, Santos EM, Jaeger MM (2001) Cytotoxic effects of $10 \%$ citric acid and EDTA-T used as root canal irrigants: an in vitro analysis. J Endod 27: 741-743.

34. Malheiros CF, Marques, MM, Gavini G (2003) The effect of various concentrations of sodium hypochlorite on the ability of MTAD to remove the smear layer. J Endod 29: 233-239.

35. Amaral KF, Rogero MM, Fock RA, Borelli P, Gavini G (2007) Cytotoxicity analysis of EDTA and citric acid applied on murine resident macrophages culture. Int Endod J 40: 338-343. 